\title{
Numerical analyses of JT-60SA with tungsten divertor by COREDIV code
}

\author{
K. Gałązka ${ }^{1}$, I. Ivanova-Stanik ${ }^{1}$, W. Stępniewski ${ }^{1}$ and \\ R. Zagórski ${ }^{1}$, R. Neu $^{2,3}$, M. Romanelli ${ }^{4}$, T. Nakano ${ }^{5}$ \\ 1 Institute of Plasma Physics and Laser Microfusion, Hery 23, 01-497 Warszawa, \\ Poland \\ 2 Max Planck Institut für Plasmaphysik, Boltzmannstraße 2, D-85748 Garching, \\ Germany \\ 3 Technische Universität München, Boltzmannstraße 15, 85748 Garching, \\ Germany \\ 4 CCFE, Culham Science Centre for Fusion Energy, Abingdon, Oxon, \\ OX14 3DB, United Kingdom \\ 5 National Institutes for Quantum and Radiological Science and Technology \\ 801-1, Mukoyama, Naka, Ibaraki 311-0193 JAPAN \\ E-mail: Krzysztof.Galazka@ifpilm.pl
}

\begin{abstract}
.
An analysis of radiative power exhaust for JT-60SA tokamak with tungsten divertor is performed with the help of self-consistent, core-edge integrated COREDIV code. Two scenarios of operation (low and high density) were investigated in the scope of different parameters (electron density at the separatrix and transport in the scrape-off layer) with impurity seeding (Ne and $\mathrm{Kr}$ ).

The calculations show that in case of tungsten divertor the power load to the plate is mitigated and the central plasma dilution is smaller compared to the carbon divertor. In the most cases the energy flux through the separatrix is above the L-H transition threshold. For the high density case with neon seeding operation in full detachment mode is observed. It is demonstrated that transport in the SOL has a high influence on the result of calculations, for instance by changing the electron density on the separatrix the influx of heavy impurities (W, $\mathrm{Kr}$ ) into the core region can be reduced.

Keywords: tokamak, JT-60SA, integrated MHD modeling, impurity seeding
\end{abstract}

PACS numbers: 52.55.Fa, 52.65.Kj, 28.52.Av

Submitted to: Nucl. Fusion

\section{Introduction}

The main aim of JT-60SA project is to analyze the near-fusion plasma conditions for support of ITER experiment [1,2]. The crucial problem for a future tokamak reactor is the reduction of the power delivered to divertor plates down to levels acceptable for existing tungsten-based materials. According to engineering limits for ITER, the maximum heat dissipated in the divertor plate has to be lower than $10 \mathrm{MW} / \mathrm{m}^{2}$ in steady 
state [3]. This can be achieved by radiative cooling in the pedestal and scrape-off layer (SOL) areas, which helps to spread the energy over a larger area and to reduce the energy flux towards the target. Effective radiating cooling can be provided by seeded or intrinsic impurities through various radiation channels.

The influence of various seeding gases on the improvement of energy confinement on JT-60U has been extensively investigated experimentally and numerically. For instance in Ne-seeded discharges neon was found to contribute the dominant part of radiation during detachment [4]. Moreover, discharges with argon seeding beside expected increase in radiation, especially in the vicinity of the pedestal, showed additional increase in confinement during the seeding phase $[5,6]$. The explanation of the behavior is not yet clear, but it is accompanied by density peaking and a rise in electron temperature in the pedestal region. SOL simulations with Ar seeding were also performed by SONIC code, which incorporates real geometry and Monte-Carlo algorithm [7]. However the tungsten concentration used there, which is a crucial value, is a given parameter, whereas in COREDIV it is calculated self-consistiently. In any case the results show that high seeding gas puff and fueling levels are necessary to fulfill the scenario requirements.

The analysis of the influence of different kinds of impurities (divertor material and seeded impurities) in the future device JT-60SA is performed by using a numerical code COREDIV [8]. This integrated core-edge fluid code can provide steady-state impurity concentrations and radiation distributions taking into account basic atomic processes (radiation, recombination, charge exchange) and plasma-wall interactions. COREDIV is able to simulate tokamak plasmas with various divertor plate materials as well as various plasma impurities $[9,10,11]$. In our previous work we analyzed scenarios for JT-60SA with a carbon divertor [12]. Until recently carbon was considered as a perspective first wall material, but experiments show that besides numerous advantages, due to chemical activity (reaction with tritium) it should not be used in future tokamak reactors [3, 13]. Therefore, according to JT-60SA Research Plan the carbon wall will be exchanged with tungsten [1]. Tungsten is considered the most suitable plasma facing material du to several beneficial properties: high melting point, no chemical erosion, high sputtering threshold energy for deuterium, moderate activation [3, 13]. In burning plasmas radiating tungsten ions can more effectively remove the excess energy from the core region than $\mathrm{C}$ and do not cause strong plasma dilution. On the other hand, due to high $Z$ very little concentration of tungsten in the core are allowed before causing a radiative collapse [14]. Therefore, using seeded impurities to cool down the SOL and to further limit tungsten sputtering is crucial for stable operation of ITER and larger fusion devices. As due to high $Z$ tungsten radiates mostly in the core, where the temperatures are higher, enhancing the radiation in the the pedestal and SOL region requires the seeding of a single low- $Z$ or a mixture of low- and medium- $Z$ impurities $[15,16]$. Last but not least, to achieve high density regime with detached divertor a substantial amount of energy needs to be dissipated in the SOL region, which is facilitated by using externally seeded impurities.

\subsection{Code description}

The approach of solving the plasma equations by dividing the tokamak into a unidimensional core and at least 2 dimensional SOL is a known method [17, 18]. In COREDIV the SOL is described by $2 \mathrm{D}$ MHD multi-fluid equations developed by Braginskii [19]. Transport along the magnetic filed lines is assumed to be 
classical, whereas the perpendicular transport is anomalous with empirical diffusion coefficients. In the SOL region continuity equation, parallel momentum transport for each ionization state and equations for electron and ion temperature (common for all ions) are solved. In the core 1D radial diffusion equations for densities of ions and electrons and their temperatures are solved. Each ionization state of each impurity is considered separately in both domains. The coupling between the core and SOL region is imposed by continuity conditions on the separatrix for values of temperatures and densities and fluxes of particles and energy. For the ion transport equations in the core region anomalous transport coefficients for densities and temperatures are used. In COREDIV the neutrals are described by a simplified analytical model based on a diffusive behavior with adjustable recycling coefficients to reproduce the assumed average electron density and its value on the separtrix. This approximation enables fast computation and retains the most of the important observations while sacrificing the description of rich details of plasma-wall interaction and behavior of neutral particles $[8,9,10]$.

The electron and ion energy fluxes are defined by the local transport model as implemented in [20] which reproduces a prescribed energy confinement law. In particular, the anomalous heat conductivity is given by the expression $\chi_{e, i}=$

$2 C_{e, i} a^{2} / \tau_{\mathrm{E}}\left[0.25+0.75(r / a)^{4}\right] F(r)$, where $\tau_{\mathrm{E}}$ is the energy confinement time defined by the ELMy H-mode IPB98 $(\mathrm{y}, 2)$ scaling law [21], $a$ is the minor plasma radius and the energy transport coefficients $C_{e}, C_{i},\left(C_{e}=C_{i}\right)$ are adjusted to have agreement between the calculated and experimental confinement times. Transport coefficients have been modified in the pedestal region by a peak-shaped function $F(r)$ to model the transport barrier with an extreme positioned at $\rho_{\text {pol }} / a=0.93$, where $\rho_{\text {pol }}$ is the poloidal coordinate. The anomalous radial diffusion coefficients for electrons and ions in the core $D_{e, i}^{a n}=0.1 \chi_{e, i}$, respectively. The impurity concentration is defined as the ratio of impurity density to the electron density.

In COREDIV the description of the transport in the core region is simplified as the main aim is to analyze the influence of the impurities on the radiative heat losses, divertor target load and tungsten concentration. The simplifications allow to avoid time-consuming sophisticated transport models and Monte-Carlo calculations. However, one has to take into account the limitations of the model resulting from the assumptions, for instance no implemented treatment of ELM's. Therefore, all the COREDIV results should be interpreted as time-averaged quantities [10].

\subsection{Modeled scenarios}

In our previous work we analyzed JT-60SA inductive scenarios \#2 (low density, high auxiliary power, referred to as \#2) and \#3 (high density, low auxiliary power, referred to as \#3) for the configuration with carbon divertor and 4 impurities: nitrogen, neon, argon and krypton [12]. For scenario details see Table 1.1 [1]. Although for \#2 it was not possible to limit the high power load on the divertor plate despite various seeding impurities, krypton was suggested for further study as it leads to beneficial conditions in the divertor. The present work focuses on the calculations for the same scenarios, but with tungsten divertor. The main focus is to study the stationary state achieved for different gas seeding levels. Moreover, the influence of model parameters (radial diffusion coefficient in the SOL and electron density at the separatrix) on the stationary state are analyzed. In particular, the total impurity concentrations, radiation losses and corresponding heat loads at the target are calculated. Here neon 
Numerical analyses of JT-60SA with tungsten divertor by COREDIV code

\begin{tabular}{lr|cc} 
Parameter name & Unit & Scn\#2 & Scn\#3 \\
\hline$I_{\mathrm{p}}$ & {$[\mathrm{MA}]$} & 5.5 & 5.5 \\
$B_{\mathrm{T}}$ & {$[\mathrm{T}]$} & 2.25 & 2.25 \\
$R_{\mathrm{T}} / \mathrm{a}$ & {$[\mathrm{m}]$} & $2.96 / 1.18$ & $2.96 / 1.18$ \\
$\kappa$ & {$[-]$} & 1.87 & 1.86 \\
$P_{\text {aux }}$ & {$[\mathrm{MW}]$} & 41 & 30 \\
$\left\langle n_{\mathrm{e}}\right\rangle_{\text {LIN }} /\left\langle n_{\mathrm{e}}\right\rangle_{\text {VoL }} / n_{\mathrm{e}}(0)$ & {$\left[\times 10^{19} \mathrm{~m}^{-3}\right]$} & $6.3 / 5.6 / 7.7$ & $10 / 9 / 12.3$ \\
$\left\langle T_{\mathrm{e}}\right\rangle_{\text {VOL }} / T_{\mathrm{e}}(0)$ & {$[\mathrm{keV}]$} & $6.3 / 13.5$ & $3.7 / 7.9$ \\
$\left\langle T_{\mathrm{i}}\right\rangle_{\text {VOL }} / T_{\mathrm{i}}(0)$ & {$[\mathrm{keV}]$} & $6.3 / 13.5$ & $3.7 / 7.9$ \\
$\mathrm{H}_{98(y, 2)}$ & {$[-]$} & 1.3 & 1.1
\end{tabular}

Table 1. Selected parameters of the simulated JT-60SA scenarios. From the top: plasma current, central magnetic field, totoidal/poloidal radius, elongation, maximum auxiliary heating power, electron density: linear average/volume average/central, electron temperature: volume average/central, ion temperature: volume average/central, $\mathrm{H}$-factor according to $\operatorname{IPB} 98(\mathrm{y}, 2)$ scaling law.

and krypton are selected as the examples of low- and high- $Z$ impurities. Due to the fact that in \#3 the density is higher and the auxiliary power much lower than in \#2 neon is able to provide enough cooling and simulations with krypton are omitted.

\section{Results}

In the beginning some preliminary calculations were performed to adjust the transport barrier parameters (position, height and decay length) and main plasma ion inward pinch velocity to better match the density and temperature profiles for the unseeded discharges resulting from JETTO calculations (for scn\#2) [22]. Also the ratio of auxiliary heating power delivered to ions and electrons was adjusted and set to 50/50. Then the scans of seeding gas puff rate were performed for different values of 2 adjustable parameters: radial diffusion coefficient for ions in SOL (main plasma and impurities) $D_{\perp}$ and the ratio of the electron density on the separatrix to the average electron density $n_{\mathrm{e}}^{\mathrm{sep}} /\left\langle n_{\mathrm{e}}\right\rangle$. As presented in Table 2 , the reference values of $D_{\perp}$ and $n_{\mathrm{e}}^{\mathrm{sep}} /\left\langle n_{\mathrm{e}}\right\rangle$ were chosen to $0.5 \mathrm{~m}^{2} / \mathrm{s}$ and $40 \%$, respectively. Two more values of $D_{\perp}$ $\left(0.25 \mathrm{~m}^{2} / \mathrm{s}\right.$ and $\left.1 \mathrm{~m}^{2} / \mathrm{s}\right)$ while keeping $n_{\mathrm{e}}^{\mathrm{sep}} /\left\langle n_{\mathrm{e}}\right\rangle=40 \%$ and two more $n_{\mathrm{e}}^{\text {sep }} /\left\langle n_{\mathrm{e}}\right\rangle$ values (50\% and $60 \%$ ) while keeping $D_{\perp}=0.5 \mathrm{~m}^{2} / \mathrm{s}$ were analyzed.

\begin{tabular}{|c|c|c|c|}
\hline \multirow{2}{*}{$n_{\mathrm{e}}^{\mathrm{sep}} /\left\langle n_{\mathrm{e}}\right\rangle \downarrow$} & \multicolumn{3}{|c|}{$D_{\perp} / \mathrm{m}^{2} / \mathrm{s}$} \\
\hline & 0.25 & 0.5 & 1 \\
\hline $\begin{array}{l}40 \% \\
50 \%\end{array}$ & $\checkmark$ & $\begin{array}{l}\checkmark \\
\checkmark\end{array}$ & $\checkmark$ \\
\hline $60 \%$ & & $\checkmark$ & \\
\hline
\end{tabular}

Table 2. Parameter space analyzed for each investigated scenario.

\subsection{General seeding dynamics observations}

The key to understanding further results is to recognize the influence of $D_{\perp}$ and $n_{\mathrm{e}}^{\mathrm{sep}} /\left\langle n_{\mathrm{e}}\right\rangle$ on the dynamics of the impurity transport. The sources of impurities, both intrinsic and seeded, are localized in the SOL. The seeded impurity (neon and krypton) 
is puffed at a localized spot situated at $90 \%$ of the distance along the inner wall between the stagnation point and the target. Tungsten production on the plate is determined by the sputtering coefficient, which is strongly coupled to the electron temperature at the plate $T_{\mathrm{e}}^{\mathrm{PLATE}}$ and the ion influx. The influx of impurities into the central plasma is calculated according to multi-fluid transport equations valid in the SOL. Therefore, changing $D_{\perp}$ and $n_{\mathrm{e}}^{\mathrm{sep}} /\left\langle n_{\mathrm{e}}\right\rangle$ (boundary condition) effectively changes the balance of the core-SOL system, introduces new temperature profiles and distributes all ionization states of the impurities in a different way. These changes inherently entail a change of radiation profiles, energy exhaust and conditions on the divertor target, which is of our central interest.
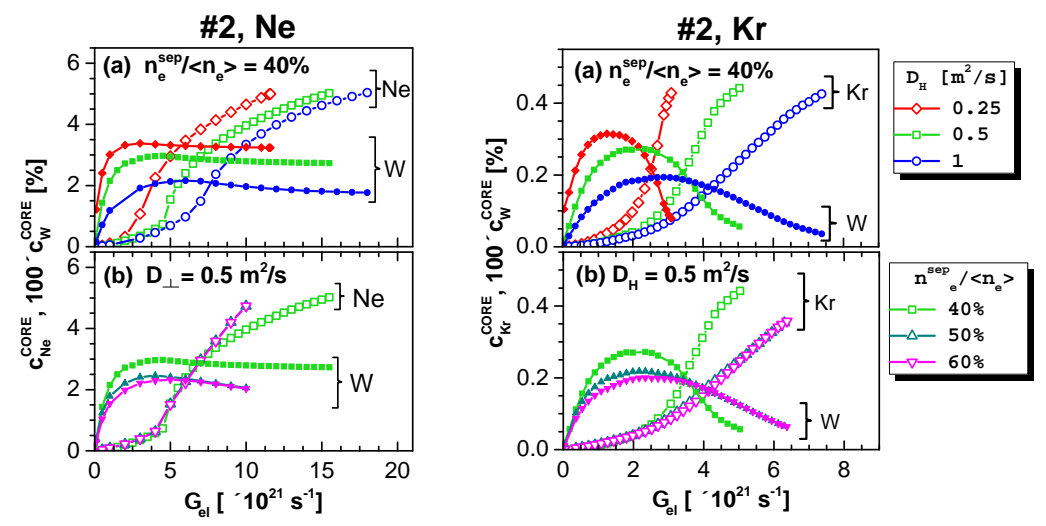

Figure 1. Concentrations of intrinsic (W, filled symbols) and seeded ( $\mathrm{Ne}, \mathrm{Kr}$, open symbols) impurities as a function of seeding for different values of $D_{\perp}$ (a) and $n_{\mathrm{e}}^{\text {sep }} /\left\langle n_{\mathrm{e}}\right\rangle$ (b) for $\# 2$. The data for the reference values of $D_{\perp}=0.5 \mathrm{~m}^{2} / \mathrm{s}$ and $n_{\mathrm{e}}^{\mathrm{sep}} /\left\langle n_{\mathrm{e}}\right\rangle=40 \%$ is shown in every graph.

In Figure 1 the core concentration of impurities $\left(c_{\mathrm{W}}^{\mathrm{CORE}}, c_{\mathrm{Ne}}^{\mathrm{CORE}}, c_{\mathrm{Kr}}^{\mathrm{CORE}}\right)$ as a function of gas puff level of the seeded impurity (seeding rate of neon and krypton recalculated to the amount of electrons per second, $\left.\Gamma_{\mathrm{el}}\right)$ for different values of $D_{\perp}$ (a) and $n_{\mathrm{e}}^{\mathrm{sep}} /\left\langle n_{\mathrm{e}}\right\rangle$ (b) are presented. Impurity concentrations achieved with neon seeding are larger by an order of magnitude than for krypton. Seeding of $\mathrm{Kr}$ is lower than for $\mathrm{Ne}$ as $\mathrm{Kr}$ has higher radiation efficiency in the core region. In the terms of discharge control and limiting the tungsten concentration it is beneficial to have higher $D_{\perp}$ and higher $n_{\mathrm{e}}^{\mathrm{sep}} /\left\langle n_{\mathrm{e}}\right\rangle$.

A general observation is that the heavier impurity $(\mathrm{Kr})$ suppresses $c_{\mathrm{w}}^{\mathrm{CORE}}$ by replacing tungsten in the core, whereas the lighter one (neon) does not. While increasing $D_{\perp}$ the growth of $c_{\mathrm{Ne}}^{\mathrm{CORE}}$ and $c_{\mathrm{Kr}}^{\mathrm{CORE}}$ with increasing gas puff is slower in both cases and the maximum achieved $c_{\mathrm{W}}^{\mathrm{CORE}}$ is suppressed. This observation can be explained by screening effect caused by increased radial transport of tungsten ions in the SOL.

As visible in Figure $1(\mathrm{~b})$, increasing $n_{\mathrm{e}}^{\mathrm{sep}} /\left\langle n_{\mathrm{e}}\right\rangle$ has a little effect on $c_{\mathrm{Ne}}^{\mathrm{CORE}}$, but the growth of $c_{\mathrm{Kr}}^{\mathrm{CORE}}$ is suppressed. Also with increasing $n_{\mathrm{e}}^{\mathrm{sep}} /\left\langle n_{\mathrm{e}}\right\rangle$ the maximum tungsten concentration is limited. Higher $n_{\mathrm{e}}^{\mathrm{sep}} /\left\langle n_{\mathrm{e}}\right\rangle$ increases the friction between impurity ions and main plasma leading to the reduction of the influx of impurity ions into the core plasma. In both cases increase of $n_{\mathrm{e}}^{\mathrm{sep}} /\left\langle n_{\mathrm{e}}\right\rangle$ above $50 \%$ brings almost no change in transport dynamics. This saturation behavior is visible in several other parameters, as demonstrated in the section 2.4. 
From the technical point of view it should be easier to control the concentration of seeded impurity in the case of neon as the gas puff range is wider than in the case of krypton and the slope of $c_{\mathrm{Ne}}^{\mathrm{CORE}}\left(\Gamma_{\mathrm{el}}\right)$ dependence is less steep. The results of calculations for \#3 are similar to the ones presented above albeit with $c_{\mathrm{W}}^{\mathrm{CORE}}$ about a factor of 10 lower, therefore are not shown.

\subsection{Electron density and temparature profiles}

Figure 2 presents the electron density $n_{e}$ (a) and electron temperature $T_{e}$ (b) profiles for the reference values of $D_{\perp}$ and $n_{\mathrm{e}}^{\text {sep }} /\left\langle n_{\mathrm{e}}\right\rangle$. In fact, the density depends very weakly on the kind of impurity and the seeding rate, mostly due to fixed conditions for $n_{\mathrm{e}}^{\mathrm{sep}} /\left\langle n_{\mathrm{e}}\right\rangle$ and $\left\langle n_{e}\right\rangle$, so the profiles presented in Figure 2 (a) refer to the whole investigated concentration range for a given scenario. But the temperature profiles show an increased peaking when the impurity concentration is increased. In Figure 2 (b) $T_{e}$ exemplary profiles for $\left\langle Z_{\text {eff }}\right\rangle=3$ for each seeding case are shown. For comparison the unseeded cases are also presented as the dashed lines. It can be noticed that in \#2 with krypton seeding the central temperature is slightly lower than with neon for the same $\left\langle Z_{\text {eff }}\right\rangle$ value due to lower dilution of the main plasma. In general, the $T_{e}$ profiles are lower compared to our previous results for carbon divertor [12], mainly due to the mentioned adjustment of barrier parameters: inward pinch velocity and transport barrier height, which were afterwards kept constant during the simulations. As-adjusted profiles have central $n_{e}(0)$ and $T_{e}(0)$ values are in very good agreement with the designed ones (see Table 1.1).

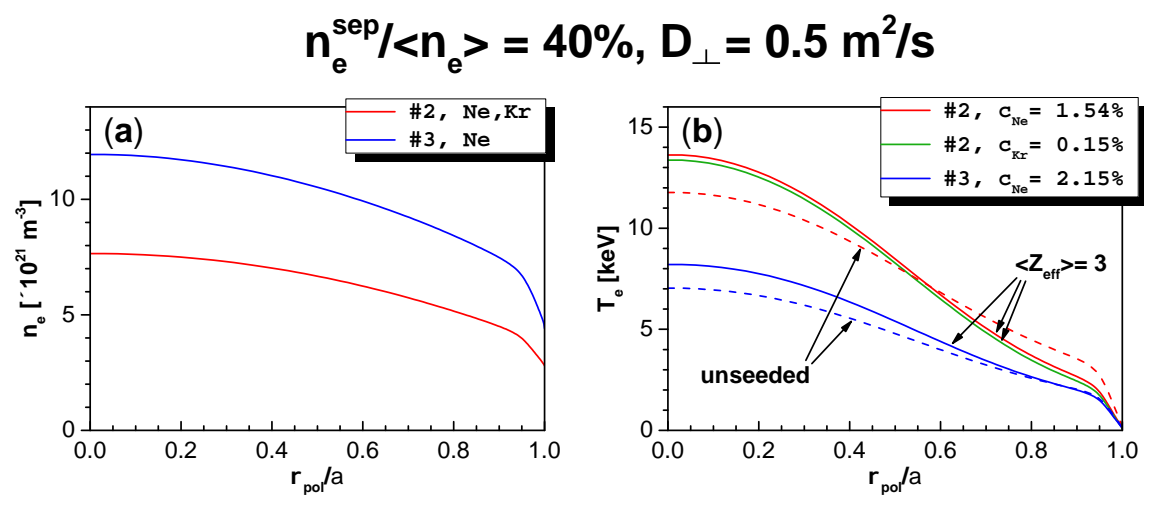

Figure 2. Poloidal profiles of electron density (a) and temperature (b) in \#2 for $D_{\perp}=0.5 \mathrm{~m}^{2} / \mathrm{s}$ and $n_{\mathrm{e}}^{\mathrm{sep}} /\left\langle n_{\mathrm{e}}\right\rangle=40 \%$. For details see text.

\subsection{The influence of different $D_{\perp}$}

This section describes the influence of radial diffusion in the SOL $D_{\perp}$ on the plasma properties. Figure 3 presents the radiation power $P_{\mathrm{RAD}}^{\mathrm{TOT}}$ (a) and $P_{\mathrm{RAD}}^{\mathrm{SOL}}$ (ac), concentration of tungsten in the core $c_{\mathrm{W}}^{\mathrm{CORE}}(\mathrm{c})$ and average effective charge $\left\langle Z_{\text {eff }}\right\rangle$ (d) as a function of seeded impurity concentration in the core.

The average effective charge is linearly dependent on the seeded impurity concentration with the slope determined by the kind of the seeded impurity and the intercept set by initial tungsten concentration when no seeding is applied. It can be 


\section{$\mathrm{n}_{\mathrm{e}}^{\mathrm{sep}} /<\mathrm{n}_{\mathrm{e}}>=40 \%$}
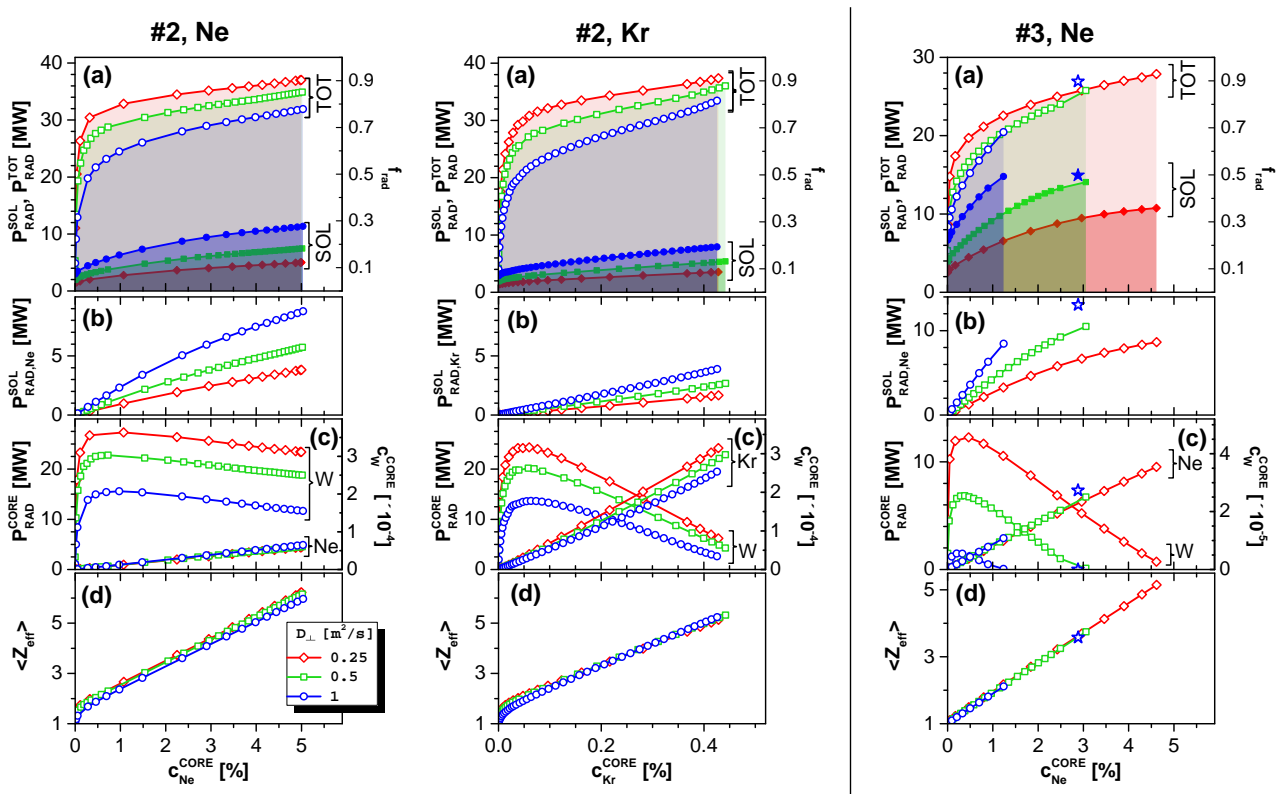

Figure 3. Radiation powers, tungsten concentration and average effective charge as a function of seeded impurity concentration for \#2 (left and middle) and $\# 3$ (right) for different values of $D_{\perp}$. Total radiation power $P_{\mathrm{RAD}}^{\mathrm{TOT}}, \mathrm{SOL}$ radiation power $P_{\mathrm{RAD}}^{\mathrm{SOL}}$ (filled symbols) and radiation fraction $f_{\mathrm{RAD}}$ (right scale) (a), radiation power of the seeded impurity in the SOL (b), radation power of the seeded impurity and tungsten in the core $P_{\mathrm{RAD}, \mathrm{Ne} / \mathrm{Kr}}^{\mathrm{CORE}}, P_{\mathrm{RAD}, \mathrm{W}}^{\mathrm{CORE}}$, respectively, and tungsten cocnentration in the core $c_{\mathrm{W}}^{\mathrm{CORE}}$ (right scale, overlaps with $P_{\mathrm{RAD}}^{\mathrm{CORE}}$ ) ) (c), average effective charge $\left\langle Z_{\text {eff }}\right\rangle$ (d). The blue star symbol in \#3 with Ne denotes the last point for the $D_{\perp}=1 \mathrm{~m}^{2} / \mathrm{s}$ series, a detached state.

observed that varying $D_{\perp}$ has a negligible influence on $\left\langle Z_{\text {eff }}\right\rangle$. It should be pointed out that for the purpose of fusion reactor it is beneficial to keep the $\left\langle Z_{\text {eff }}\right\rangle$ as low as possible to avoid nuclear fuel dilution. However, for the purpose of studying radiative exhaust by different impurities we present simulations with $\left\langle Z_{\text {eff }}\right\rangle$ up to about 5 , what corresponds to plasmas with $0.5 \%$ of $\mathrm{Kr}$ or $4 \%$ of Ne. This approach allows for observing more general trends and features of seeding with different impurities.

In the model the impurity line radiation of each specie proportional to $n_{e}^{2}$. $L^{\mathrm{Ne} / \mathrm{Kr}} \cdot c_{\mathrm{Ne} / \mathrm{Kr} / \mathrm{W}}^{\mathrm{CORE}}$ where $L^{\mathrm{Ne} / \mathrm{Kr} / \mathrm{W}}(T)$ is the respective cooling factor. In the case of seeded impurities it exhibits a linear dependence on $c_{\mathrm{Ne} / \mathrm{Kr}}^{\mathrm{CORE}}$ (see Figure 4 (c)). It is clear that as for a given scenario the electron density profile does not change with seeding, the variation of $P_{\mathrm{RAD}, \mathrm{Ne} / \mathrm{Kr}}^{\mathrm{CORE}}$ with $D_{\perp}$ is due to changes in the electron temperature profile. On the other hand for the intrinsic impurity the dominant factor ruling its $P_{\mathrm{RAD}, \mathrm{W}}^{\mathrm{CORE}}$ is $\mathrm{W}$ core concentration. The shape of $P_{\mathrm{RAD}, \mathrm{W}}^{\mathrm{CORE}}$ almost directly overlaps with $c_{\mathrm{W}}^{\mathrm{CAOE}}$ (note the right scale in Figure $4(\mathrm{c})$ ). It can be noted that increasing $D_{\perp}$ enhances the screening effect in the SOL which results in lower high- $Z$ impurities radiation in the core.

In \#2 krypton radiates in the core more effectively than neon due to higher $Z$. Moreover, it can be observed that krypton radiation replaces tungsten radiation in 
the core. This is also true for neon in \#3, due to different conditions than in \#2 (see Figure $2(\mathrm{~b}))$. Here at sufficiently high $c_{\mathrm{Ne}}^{\mathrm{CORE}}$ tungsten could be entirely replaced by Ne. The impurity concentration at which maximum $c_{\mathrm{W}}^{\mathrm{CORE}}$ occurs is $0.06 \%$ of $\mathrm{Kr}$ in $\# 2$ and $0.2-0.5 \%$ of $\mathrm{Ne}$ in \#3 (lower end for higher $D_{\perp}$ value). Exceeding these values experimentally while sustaining stable $\mathrm{H}$-mode plasma would open a way to removal of tungsten impurity and to detachment. On the other hand, it must be emphasized that high impurity concentrations correspond to high $\left\langle Z_{\text {eff }}\right\rangle$ values and might be not applicable for a reactor scenario.

Concerning the SOL radiation in the case of \#2 the seeded impurity radiation plays a dominant role, as visible when comparing Figures 3 (a) and (b). In scenarios with Ne seeding an increase in $D_{\perp}$ causes $P_{\mathrm{RAD}, \mathrm{Ne}}^{\mathrm{SOL}}$ to grow, especially in \#3. As $\mathrm{Kr}$ radiates in SOL less than Ne the influence of $D_{\perp}$ is less pronounced in \#2. On the other hand, total radiation power losses $P_{\mathrm{RAD}}^{\mathrm{TOT}}$ (and radiation fraction $f_{\mathrm{RAD}}$ ) diminish while increasing $D_{\perp}$, especially in \#2. This tendency is caused by the behavior of tungsten (Figure 4 (c)) and can be explained by the shielding effect described in Section 2.1. Tungsten transport is crucial for determining the core radiation in \#2. For the case with $\left\langle Z_{\text {eff }}\right\rangle=3$ and $D_{\perp}=0.5 \mathrm{~m}^{2} / \mathrm{s}$ the highest achieved radiation fraction is about 0.85 in \#2 for both impurities and about 0.75 for \#3. It should be noted that in the case of \#3 the detachment regime is easily accessible and for the case with $D_{\perp}=1 \mathrm{~m}^{2} / \mathrm{s}$ for $c_{\mathrm{Ne}}^{\mathrm{CORE}} \approx 1.25 \%$. Achieved $f_{\mathrm{RAD}}=0.7$ is already rather high and about $75 \%$ of the total radiation power is radiated out in the SOL (see Figure 3 (a)).

Figure 4 (a) presents the input power to the SOL $P_{\text {input, тот }}^{\text {SOL }}$ as a function of seeded impurity concentration. The dashed area denotes the L-mode regime, where the threshold value has been calculated from the condition elaborated by Martin et al. [23]. Only in the case of the lowest $D_{\perp}$ value in \#2 staying in $\mathrm{H}$-mode at high seeding rate might be an issue.

In Figure 4 (b) the total power delivered to the divertor plate $P_{\mathrm{TOT}}^{\mathrm{PLATE}}$ is presented. The limit marked by the black line corresponds to maximum average heat flux on the plate of $q_{\|, M A X}=10 \mathrm{MW} / \mathrm{m}^{2}$ [1]. It is hard to set a definite power limit in calculations with simplified geometry. In our approach the limit is calculated from $P_{\mathrm{TOT}, \mathrm{MAX}}^{\mathrm{PLATE}}$ $=q_{\|, M A X} \cdot A$, where total wetted area $A$ is estimated from $A=2 \pi R_{T} \cdot \lambda_{\text {int }} \cdot f_{x} / \sin (\alpha)$. Here the toroidal radius $R_{T}$ is an estimate of the divertor radius, $\lambda_{\text {int }}$ is the integral power fall-off length of the heat flux profile defined as in [24], $f_{x}$ is the effective flux expansion coefficient and $\alpha$ is the angle formed between magnetic field lines and the divertor tiles in the poloidal cross-section $\left(\alpha=45^{\circ}\right.$, see [1]). The integral power falloff length is approximated by $\lambda_{\text {int }} \approx \lambda_{q}+1.64 s$, where the $\lambda_{q}$ is upstream power fall-off length at the midplane and $s$ is the power spreading parameter dependent on local divertor plasma parameters and geometry, characterizing the diffusion into the private region [24]. In former calculations performed for \#2 with argon at $<n_{e}>_{V O L}=9 \cdot 10^{19} \mathrm{~m}^{-3}$ the value $f_{x}=7.9$ was found and the fitting of the heat profile resulted in $\lambda_{q}=10 \mathrm{~mm}$ and $s=3 \mathrm{~mm}$ [7]. Our calculations result in $\lambda_{q}=3 \mathrm{~mm}$ or larger for \#2 and at least $\lambda_{q}=6.5 \mathrm{~mm}$ for \#3 (private region is not included in our model). Eich's scaling law gives much smaller $\lambda_{q} \in\{1.3,1.6\} \mathrm{mm}$ for all cases. If the empirical finding for JET and ASDEX-Upgrade $s / \lambda_{q}=0.42$ is assumed for JT-60SA the power spreading parameter $s$ can be estimated as at least $s=1.25 \mathrm{~mm}$ for $\# 2$ and at least $s=2.7 \mathrm{~mm}$ for \#2. The wetted area resulting from these approximations is $0.8 \mathrm{~m}^{2}$ up to few $\mathrm{m}^{2}$. The maximum total divertor power limits shown in Figure 4 (b) are caluclated assuming the same $f_{x}=7.9$ as in case of Ar. It must be emphasized that in real geometry there are two divertors, inner and outer and the plotted $P_{\mathrm{TOT}}^{\text {PLATE }}$ is 


\section{$n_{e}^{\text {sep }} /<n_{e}>=40 \%$}
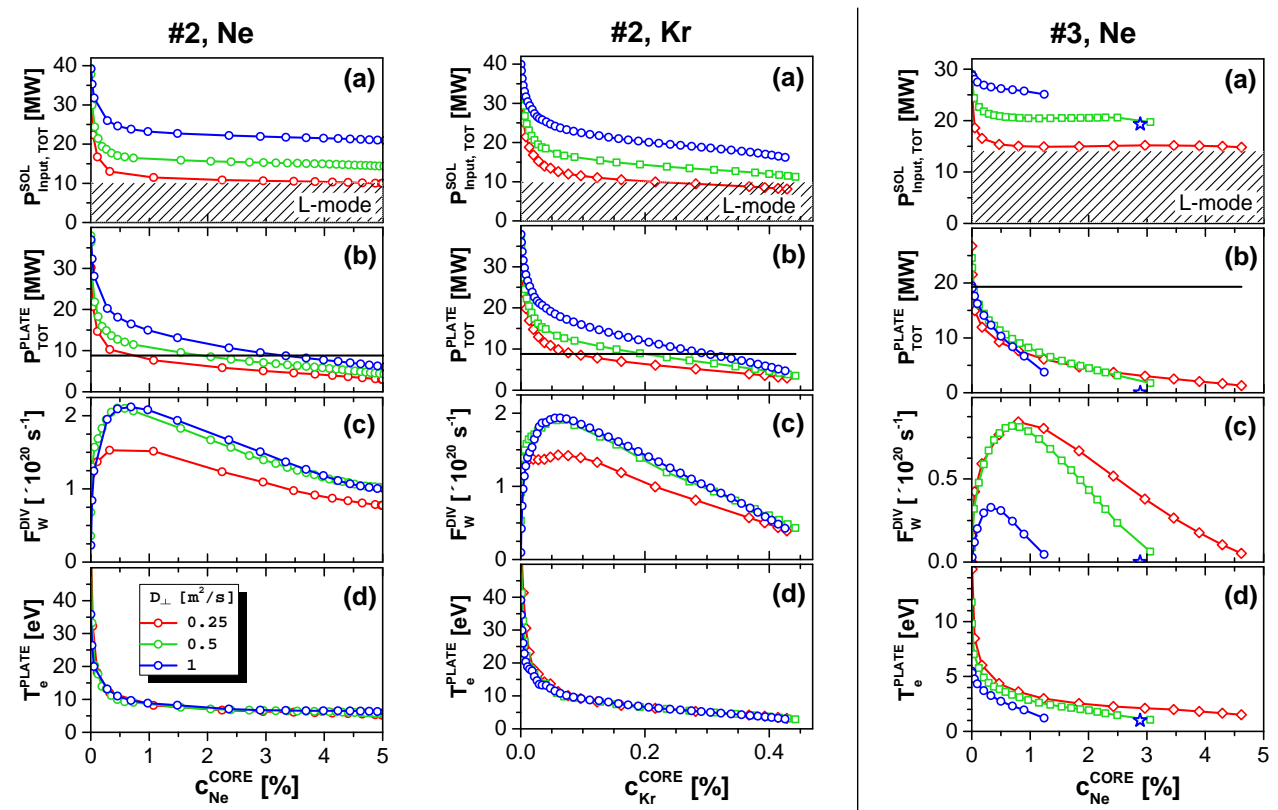

Figure 4. Physical parameters of the SOL region and divertor plate as a function of seeded impurity concentration for \#2 (left and middle) and \#3 (right) for different values of $D_{\perp}$ : input power to the SOL $P_{\text {input, тOT }}^{\mathrm{SOL}}$ (a) (L-mode regime is marked with dashed lines), total power delivered to the divertor plate $P_{\mathrm{TOT}}^{\mathrm{PLATE}}$ (b), tungsten flux from the divertor plate $F_{\mathrm{W}}^{\mathrm{DIV}}$ (c), electron temperature at the divertor plate $T_{\mathrm{e}}^{\text {PLATE }}(\mathrm{d})$. The blue star symbol in \#3 with neon denotes the last point for the $D_{\perp}=1 \mathrm{~m}^{2} / \mathrm{s}$ series, a detached state. The black line in (b) corresponds to maximum heat flux of $q_{\|, M A X}=10 \mathrm{MW} / \mathrm{m}^{2}$.

actually delivered to a larger surface. Since in our calculations a symmetrical situation is assumed we cannot predict the precise balance of the power load of each of the targets.

All simulations start with $P_{\mathrm{TOT}}^{\text {PLATE }}$ high above the accepted upper heat flux limit. Seeding allows for reduction of the power delivered to the plate. In the case of \#3 already about $0.04 \%$ of neon is enough to suppress $P_{\mathrm{TOT}}^{\mathrm{PLATE}}$ below the limit and there is almost no influence of $D_{\perp}$ on $P_{\mathrm{TOT}}^{\text {PLATE }}$. In the case of \#2 there is a significant influence of $D_{\perp}$ on the impurity concentration at which $P_{\text {TOT }}^{\text {PLATE }}$ is below the upper accepted value: lower the $D_{\perp}$ the lower is the impurity concentration necessary for getting sufficiently low heat flux. For instance if $D_{\perp}=1 \mathrm{~m}^{2} / \mathrm{s}$ the $c_{\mathrm{Ne}}^{\mathrm{CORE}}=3.2 \%$ or $c_{\mathrm{Kr}}^{\mathrm{CORE}}$ $=0.30 \%$, both corresponding to $\left\langle Z_{\text {eff }}\right\rangle$ above 4 . In any case, using a seeding gas is compulsory for \#2 to prevent damaging the target plate.

The resulting electron temperature on the divertor plate $T_{\mathrm{e}}^{\text {PLATE }}$ values reach about $10 \mathrm{eV}$ in \#2 regardless of the seeded impurity species and falls below $5 \mathrm{eV}$ in \#3. As mentioned before, this determines the sputtering regime in the divertor and directly results in higher tungsten fluxes $F_{\mathrm{w}}^{\text {DIV }}$ in \#2 than in \#3 (Figure 4 (c). As a consequence tungsten concentrations observed in \#2 are an order of magnitude larger than in \#3. It can be noted that variation of the diffusion coefficient has almost no 
influence on $T_{\mathrm{e}}^{\text {PLATE }}$ in \#2, whereas in \#3 the $T_{\mathrm{e}}^{\text {PLATE }}$ (and $F_{\mathrm{w}}^{\text {DIV }}$ ) is suppressed when $D_{\perp}$ is increased. On the contrary, in \#2 the target temperature is not low enough and increased $D_{\perp}$ leads to even higher tungsten fluxes from the divertor.

Low $T_{\mathrm{e}}^{\text {PLATE }}$ in \#3 makes it more favorable for utilization in experiment as achieving the regime seems very plausible - the last point in the calculated data series for $D_{\perp}=1 \mathrm{~m}^{2} / \mathrm{s}$ is in full detachment.

\section{$D_{\perp}=0.5 \mathrm{~m}^{2} / \mathrm{s}$}
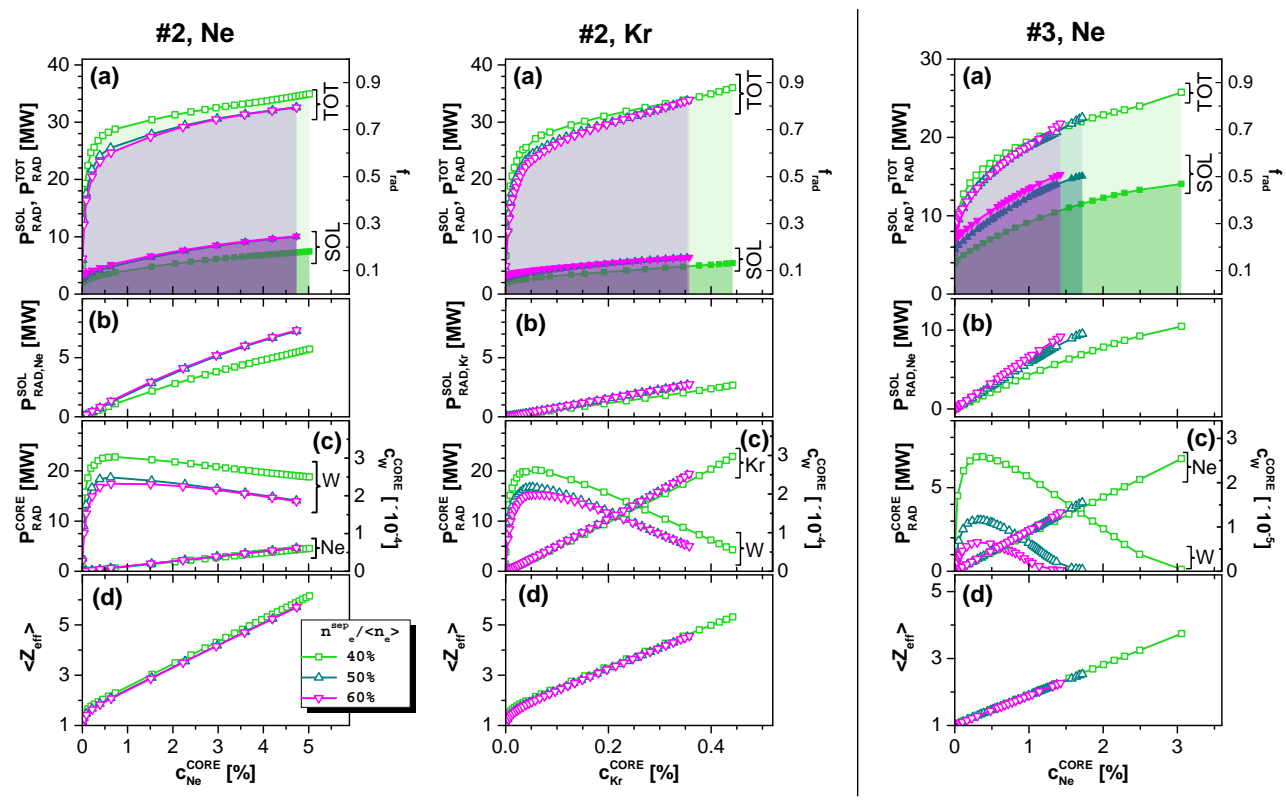

Figure 5. Radiation powers, tungsten concentration and average effective charge as a function of seeded impurity concentration for \#2 (left and middle) and \#3 (right) for different values of $D_{\perp}$. Total radiation power $P_{\mathrm{RAD}}^{\mathrm{TOT}}$, SOL radiation power $P_{\mathrm{RAD}}^{\mathrm{SOL}}$ (filled symbols) and radiation fraction $f_{\mathrm{RAD}}$ (right scale) (a), radiation power of the seeded impurity in the SOL (b), radiation power of the seeded impurity and tungsten in the core $P_{\mathrm{RAD}, \mathrm{Ne} / \mathrm{Kr}}^{\mathrm{CORE}}, P_{\mathrm{RAD}, \mathrm{W}}^{\mathrm{CORE}}$, respectively, and tungsten concentration in the core $c_{\mathrm{W}}^{\mathrm{CORE}}$ (right scale, overlaps with $P_{\mathrm{RAD}}^{\mathrm{CORE}}$ ) $(\mathrm{c}$ ), average effective charge $\left\langle Z_{\text {eff }}\right\rangle$ (d). The blue star symbol in \#3 with Ne denotes the last point for the $D_{\perp} \stackrel{\text { eff }}{=} 1 \mathrm{~m}^{2} / \mathrm{s}$ series, a detached state. The green square symbols are repeated from Figure 3 .

\subsection{The influence of different $n_{e}^{\text {sep }} /\left\langle n_{e}\right\rangle$}

In this part the influence of different $n_{\mathrm{e}}^{\mathrm{sep}} /\left\langle n_{\mathrm{e}}\right\rangle$ values is analyzed while keeping constant $D_{\perp}=0.5 \mathrm{~m}^{2} / \mathrm{s}$. Similarily as in Section 2.3, $\left\langle Z_{\text {eff }}\right\rangle$ presented in Figure 5 (d) and impurity core radiation (Figure 5 (c) exhibit a linear dependence on $c_{\mathrm{Ne} / \mathrm{Kr}}^{\mathrm{CORE}}$. As in previous case, different $n_{\mathrm{e}}^{\mathrm{sep}} /\left\langle n_{\mathrm{e}}\right\rangle$ values do not affect $\left\langle Z_{\mathrm{eff}}\right\rangle$ shape, which is determined only by seeding gas impurity concentration.

The shape of tungsten radiation almost exactly resembles its core concentration $c_{\mathrm{W}}^{\mathrm{CORE}}$, as before (Figure $5(\mathrm{c})$ ). By increasing $n_{\mathrm{e}}^{\mathrm{sep}} /\left\langle n_{\mathrm{e}}\right\rangle$ the concentration $c_{\mathrm{W}}^{\mathrm{CORE}}$ is suppressed, especially in \#3. A similar effect is visible for \#2, however beyond 
$n_{\mathrm{e}}^{\mathrm{sep}} /\left\langle n_{\mathrm{e}}\right\rangle=50 \%$ the saturation effect described in Section 2.1 takes over. This saturation concerns also several other properties, like the $P_{\mathrm{RAD}, \mathrm{Ne} / \mathrm{Kr}}^{\mathrm{SOL}}$ and power losses in Figure 5 (a) and (b), especially in \#2. In each case while $n_{\mathrm{e}}^{\text {sep }} /\left\langle n_{\mathrm{e}}\right\rangle$ is increased from $40 \%$ to $50 \%$ the total radiated power presented in Figure 5 slightly diminishes and the SOL radiation increases. It can be observed that the main source of radiation losses in the core is tungsten radiation (for details see profiles in Figure 7 (a-c)) and it rules the mentioned drop in the $P_{\mathrm{RAD}}^{\mathrm{TOT}}$. Only in \#2 with krypton and \#3 with neon at high $c_{\mathrm{Ne} / \mathrm{Kr}}^{\mathrm{CORE}}$ it is replaced by the seeded impurity radiation. However, in \#3 core radiation is much lower than in $\# 2$, as a result of one order of magnitude lower $c_{\mathrm{W}}^{\mathrm{CORE}}$. It should be pointed out again, only solutions with relatively low $\left\langle Z_{\text {eff }}\right\rangle$ are of practical interest for fusion.

\section{$D_{\perp}=0.5 \mathrm{~m}^{2} / \mathrm{s}$}

\#2, $\mathrm{Ne}$

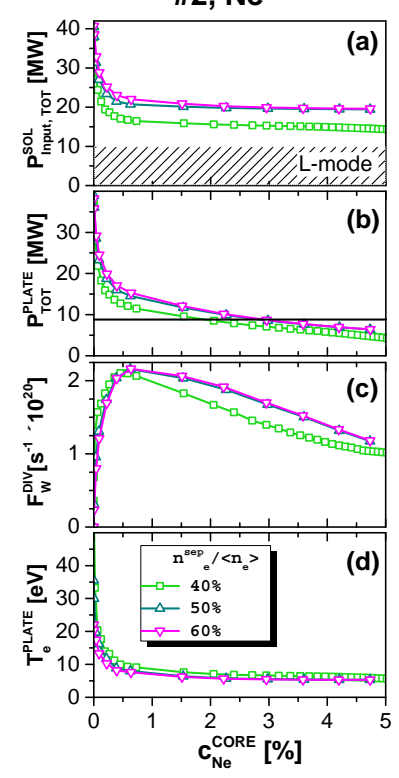

\#2, $\mathrm{Kr}$

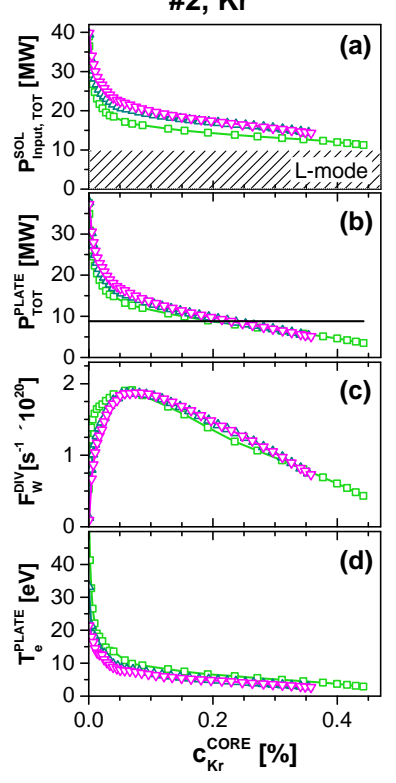

$\# 3, \mathrm{Ne}$

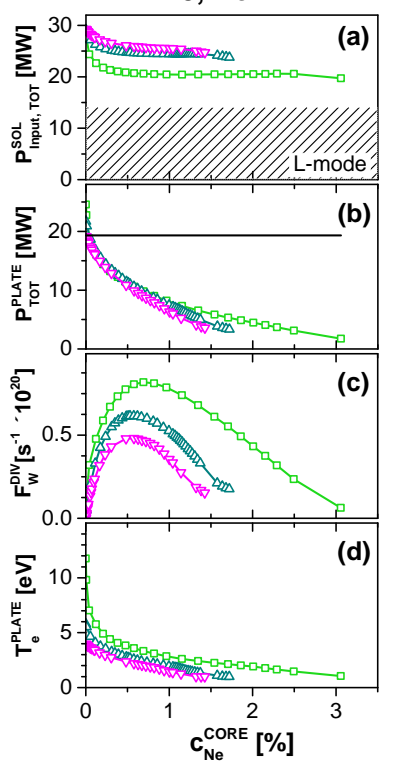

Figure 6. Physical parameters of the SOL region and divertor plate as a function of seeded impurity concentration for \#2 (left and middle) and \#3 (right) for different values of $n_{\mathrm{e}}^{\mathrm{sep}} /\left\langle n_{\mathrm{e}}\right\rangle$ : input power to the SOL $P_{\text {input, тот }}^{\mathrm{SOL}}$ (a) (L-mode regime is marked with dashed lines), total power delivered to the divertor plate $P_{\mathrm{TOT}}^{\mathrm{PLATE}}(\mathrm{b})$, tungsten flux from the divertor plate $F_{\mathrm{W}}^{\mathrm{DIV}}(\mathrm{c})$, electron temperature at the divertor plate $T_{\mathrm{e}}^{\text {PLATE }}(\mathrm{d})$. The green square symbols are repeated from Figure 4 . The black line in (b) corresponds to maximum heat flux of $q_{\|, M A X}=$ $10 \mathrm{MW} / \mathrm{m}^{2}$.

In Figure 6 (a) input power to SOL is presented. It can be seen that for operation in $\mathrm{H}$-mode it is better to have higher electron density at the separatrix. Increased $n_{\mathrm{e}}^{\mathrm{sep}} /\left\langle n_{\mathrm{e}}\right\rangle$ does not affect much the power delivered to the plate $P_{\mathrm{TOT}}^{\text {PLATE }}$, only in \#2 with neon there is a small change. However, for both impurities in \#2 it is difficult to keep $P_{\text {TOT }}^{\text {PLATE }}$ below the displayed limit of $10 \mathrm{MW} / \mathrm{m}^{2}$ for $\left\langle Z_{\text {eff }}\right\rangle$ values below $3-3.5$. The tungsten influx is almost not affected for \#2, but is suppressed in \#3 for higher values of $n_{\mathrm{e}}^{\mathrm{sep}} /\left\langle n_{\mathrm{e}}\right\rangle$ due to a similar reason: lower $T_{\mathrm{e}}^{\text {PLATE }}$. These observations are similar as 
in the case of increasing $D_{\perp}$ and the same conclusions follow.

\section{Discussion}

The results for \#3 are very promising. Already for low seeding and $c_{\mathrm{Ne}}^{\mathrm{CORE}}$ the achieved radiation fractions $f_{\mathrm{RAD}}$ are above $70 \%$, regardless the $D_{\perp}$ and $n_{\mathrm{e}}^{\mathrm{sep}} /\left\langle n_{\mathrm{e}}\right\rangle$ values. At the same time the core tungsten radiation (and concentration) is replaced by neon radiation. The SOL radiation is relatively large and originates mainly from the neon impurity, as presented in Figure 7 (c). This results in relatively large $\lambda_{q}$ values giving large wetted area and $P_{\mathrm{TOT}}^{\text {PLATE }}$ falling easily below the limit of $10 \mathrm{MW} / \mathrm{m}^{2}$ and $T_{\mathrm{e}}^{\text {PLATE }}$ below $5 \mathrm{eV}$. By increasing $D_{\perp}$ and $n_{\mathrm{e}}^{\mathrm{sep}} /\left\langle n_{\mathrm{e}}\right\rangle$ the suppression of the tungsten core concentration is stronger. At the same time the possibility of operation in H-mode is confirmed for all values of studied parameter space, except for $D_{\perp}=0.25 \mathrm{~m}^{2} / \mathrm{s}$, which leads to higher core radiation bringing the scenario close to the $\mathrm{L}-\mathrm{H}$ transition condition (see Figure 4 (a). Summarizing, the calculation results support the wide operational window concerning the external gas puff for $\# 3$. To emphasize this fact it can be mentioned that the last point in the $D_{\perp}=1 \mathrm{~m}^{2} / \mathrm{s}$ scan is in full detatchment regime (a blue star symbol for \#3 in Figures 3 and 4 ).

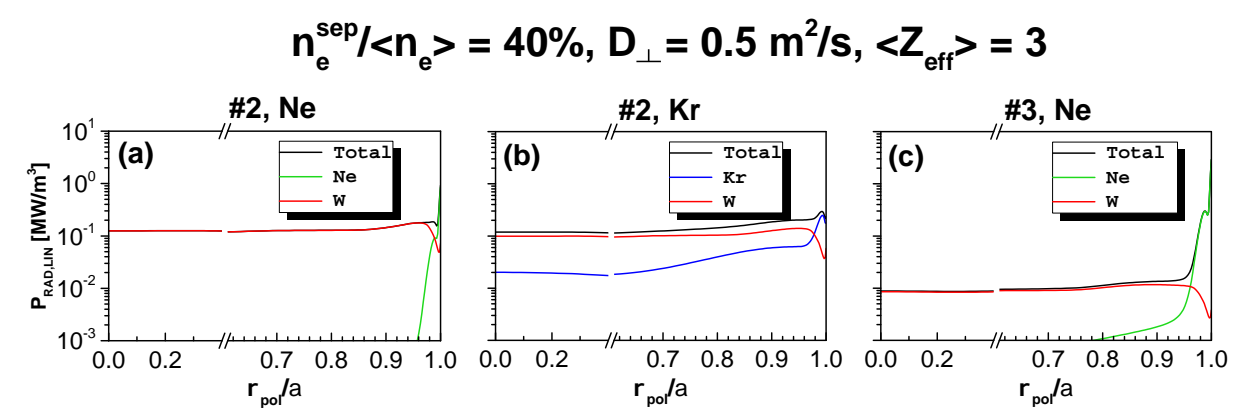

Figure 7. Line radiation from impurities for the reference case $\left(D_{\perp}=0.5 \mathrm{~m}^{2} / \mathrm{s}\right.$, $\left.n_{\mathrm{e}}^{\mathrm{sep}} /\left\langle n_{\mathrm{e}}\right\rangle=40 \%\right)$ and $\left\langle Z_{\mathrm{eff}}\right\rangle$ values around 3$)$ : \#3 with Ne seeding (a), \#2 with Ne seeding (b) and \#2 with $\mathrm{Kr}$ seeding (c), Please note a break in the abscissa for values between 0.3 and 0.6 .

In \#2 with neon seeding it is found that neon does not replace tungsten in the core, as could be seen in Figure 3 (c). Due to $c_{\mathrm{W}}^{\mathrm{CORE}}$ one order of magnitude larger than in \#3 the dominant mechanism of radiative energy loss in the core is tungsten radiation, even at high neon seeding levels. Neon radiation is concentrated in the outermost part of the plasma core and in the SOL (Figure 7 (a)). Although higher $f_{\mathrm{RAD}}$ are reached and the plasma dilution is lower than in the case with carbon divertor [12], the power delivered to the plate can arguably stay below $10 \mathrm{MW} / \mathrm{m}^{2}$. It should be emphasized that the presented $P_{\mathrm{TOT}}^{\mathrm{PLATE}}$ values are to be treated as time-averaged and do not take into account quick transients like ELM's. Operation in H-mode is possible, however becomes questionable for $D_{\perp}=0.25 \mathrm{~m}^{2} / \mathrm{s}$, similarly as in \#3. Resulting $T_{\mathrm{e}}^{\text {PLATE }}$ reaches $\sim 10 \mathrm{eV}$ and achieving the detachment regime with neon seeding seems to be impossible. Neon radiation is too low to provide enough plasma cooling.

The situation is improved by replacing low- $Z$ neon in \#2 by krypton. As can be seen in Figure 7 (b), krypton radiation replaces partially tungsten in the core and has a wide peak around the pedestal region and inside the separatrix. In the case with 
$\left\langle Z_{\text {eff }}\right\rangle=3$ krypton provides about $15 \%$ of the total radiation losses and this number grows when increasing $D_{\perp}$ and $n_{\mathrm{e}}^{\mathrm{sep}} /\left\langle n_{\mathrm{e}}\right\rangle$. Interestingly, at sufficiently high krypton seeding level it is possible to achieve $c_{\mathrm{w}}^{\text {CORE }}$ values below $10^{-4} \mathrm{~m}^{-3}$. Despite these advantages providing sufficient cooling for the target would require large gas puff and would cause significant dilution of the main plasma. The requirement of maximum $10 \mathrm{MW} / \mathrm{m}^{2}$ of power delivered to the divertor plate is met for lower gas puff when the diffusion coefficient int the SOL is lower. Similar $T_{\mathrm{e}}^{\text {PLATE }}$ values as in the case of neon seeding can be reached. The situation might be ameliorated by limiting the input power, as was proposed in our previous work [12]. On the other hand, the control of the discharge might be technically more difficult with krypton seeding than with neon as the allowable concentration range is much smaller (due to $Z$ ). Also krypton might present bigger difficulties to work in H-mode than $\mathrm{Ne}$ if $D_{\perp}$ value is low.

Although $D_{\perp}$ is a physical parameter that cannot be directly controlled in the experiment and preparing calculations with different $D_{\perp}$ values has only informative character the simulation of the influence of $n_{\mathrm{e}}^{\mathrm{sep}} /\left\langle n_{\mathrm{e}}\right\rangle$ on the scenario parameters gives some hint on experiment execution. In each case increased $n_{\mathrm{e}}^{\mathrm{sep}} /\left\langle n_{\mathrm{e}}\right\rangle$ has beneficial influence on conditions in SOL (lower $T_{\mathrm{e}}^{\mathrm{PLATE}}$, increased $P_{\text {input, тот }}^{\mathrm{SOL}}$ ) and lowers $c_{\mathrm{W}}^{\mathrm{CORE}}$ presenting a shielding effect. As an improved shielding for increased $n_{\mathrm{e}}^{\mathrm{sep}} /\left\langle n_{\mathrm{e}}\right\rangle$ values was confirmed, it provides a way to better control the behavior of the discharge, for example by increased fueling. The effectiveness of increasing $n_{\mathrm{e}}^{\mathrm{sep}} /\left\langle n_{\mathrm{e}}\right\rangle$ saturates at some point, in current calculations between $n_{\mathrm{e}}^{\mathrm{sep}} /\left\langle n_{\mathrm{e}}\right\rangle=50 \%$ and $60 \%$.

\section{Conclusions}

Two scenarios of operation of JT-60SA superconducting tokamak (\#2 with higher power and low density and \#3 with lower power and high density) were analyzed using different simulation parameters $\left(D_{\perp}\right.$ and $\left.n_{\mathrm{e}}^{\mathrm{sep}} /\left\langle n_{\mathrm{e}}\right\rangle\right)$ with seeded impurities neon and krypton (only \#2) with the help of the self-consistent, integrated COREDIV code. The calculations were based on the designed scenario parameters and resulted in electron temperature and density profiles, impurity radiation and concentration profiles and heat load of the target plate.

It was found that in \#2 the power delivered to the divertor plate is very high and with currently assumed auxiliary heating power (41 MW) using a seeding gas for controlled energy exhaust is unavoidable. Even with seeding the heat load of the target remains at levels in order of about $10 \mathrm{MW}$ with $T_{\mathrm{e}}^{\text {PLATE }}$ values about $5 \mathrm{eV}$. For a large range of examined parameters this value is above the limit of $10 \mathrm{MW} / \mathrm{m}^{2}$ and only high seeding gives possibility to protect the target plate from overheating. Using krypton as a seeding gas has an advantage that it replaces tungsten in the central plasma and limits its concentration. However, reducing the thermal load of the plate in \#2 comes at the cost of increased main plasma dilution. This point makes the operating window in \#2 quite narrow, although still open for high $\left\langle Z_{\text {eff }}\right\rangle$ cases.

In \#3 the performed simulations confirmed the existence of an operating window with beneficial conditions in the divertor, similarly to the results obtained previously for the carbon divertor configuration. It is suggested that in any case increased $n_{\mathrm{e}}^{\mathrm{sep}} /\left\langle n_{\mathrm{e}}\right\rangle$ leads to improvement of shielding and prevents tungsten and heavy impurities from entering the plasma central region. As power delivered to the SOL $P_{\text {input, тот }}^{\mathrm{SOL}}$ increases with increasing $D_{\perp}$ and radiation fraction $f_{\mathrm{RAD}}$ shows an opposite tendency, sustaining the H-mode operation might mean operating at lower radiation fraction. In $\# 3$ the power delivered to the divertor plate can be reduced with a 
minute amount of seeded impurity allowing for low dilution operation, relevant for fusion research.

\section{Acknowledgments}

This work has been carried out within the framework of the EUROfusion Consortium and has received funding from the Euratom research and training programme 20142018 under grant agreement No 633053. The views and opinions expressed herein do not necessarily reflect those of the European Commission. This scientific work was partly supported by Polish Ministry of Science and Higher Education within the framework of the scientific financial resources in the year 2016 allocated for the realization of the international co-financed project.

The authors gratefully acknowledge members of JT-60SA Integrated Project Team for data exchange and fruitful discussions.

[1] M. Yoshida and G. Giruzzi. JT-60SA Research Plan.

[2] S. Ishida, P. Barabaschi, Y. Kamada, and the JT-60SA Team. Overview of the JT-60SA Project. In 23. IAEA Fusion Energy Conference. International Atomic Energy Agency (IAEA), 2010.

[3] ITER Physics Expert Group on Divertor, ITER Physics Expert Group on Divertor Modelling, and Database and ITER Physics Basis Editors. Chapter 4: Power and particle control. Nuclear Fusion, 39(12):2391, 1999.

[4] T. Nakano, N. Asakura, and H. Kubo. Contribution of Ne ions to radiation enhancement in JT-60U divertor plasmas. Journal of Nuclear Materials, 438, Supplement:S291 - S296, 2013.

[5] H. Kubo, S. Sakurai, N. Asakura, S. Konoshima, H. Tamai, S. Higashijima, A. Sakasai, H. Takenaga, K. Itami, K. Shimizu, T. Fujita, Y. Kamada, Y. Koide, H. Shirai, T. Sugie, T. Nakano, N. Oyama, H. Urano, T. Ishijima, K.W. Hill, D.R. Ernst, A.W. Leonard, and JT60 Team. High radiation and high density experiments in JT-60U. Nuclear Fusion, 41(2):227, 2001.

[6] H. Urano, M. Nakata, N. Aiba, H. Kubo, M. Honda, N. Hayashi, M. Yoshida, Y. Kamada, and the JT-60 Team. Roles of argon seeding in energy confinement and pedestal structure in JT-60U. Nuclear Fusion, 55(3):033010, 2015.

[7] H. Kawashima, K. Shimizu, K. Hoshino, T. Nakano, and N. Asakura. Simulation of radiative divertor plasmas by Ar seeding with the full W-wall in JT-60SA. Contributions to Plasma Physics, 56(6-8):778-783, 2016.

[8] R. Stankiewicz and R. Zagórski. Influence of core-edge coupling and impurities on the operation regimes of a fusion reactor. Journal of Nuclear Materials, 337?339:191 - 195, 2005. PSI-16.

[9] R. Zagórski, G. Telesca, G. Arnoux, M. Beurskens, W. Fundamenski, and K. McCormick. Selfconsistent modeling of impurity seeded JET advanced tokamak scenarios. Journal of Nuclear Materials, 390?391:404 - 407, 2009.

[10] G. Telesca, R. Zagórski, S. Brezinsek, W. Fundamenski, C. Giroud, G. Maddison, M. O' Mullane, J. Rapp, M. Stamp, G. Van Oost, and JET EFDA contributors. Simulation with the COREDIV code of nitrogen-seeded H-mode discharges at JET. Plasma Physics and Controlled Fusion, 53(11):115002, 2011.

[11] R. Zagórski, R. Neu, and ASDEX Upgrade Team. Integrated Modelling of ASDEX Upgrade Nitrogen Seeded Discharges. Contributions to Plasma Physics, 52(5-6):379-383, 2012.

[12] R. Zagórski, G. Giruzzi, K. Gałązka, I. Ivanova-Stanik, M. Romanelli, and W. Stępniewski. Numerical analyses of JT-60SA scenarios with the COREDIV code. Nuclear Fusion, 56(1):016018, 2016.

[13] R. Neu. Tungsten as a plasma facing material in fusion devices.

[14] T. Puetterich, R. Neu, R. Dux, A.D. Whiteford, M.G. O'Mullane, H.P. Summers, and the ASDEX Upgrade Team. Calculation and experimental test of the cooling factor of tungsten. Nuclear Fusion, 50(2):025012, 2010.

[15] N. Asakura, T. Nakano, N. Oyama, T. Sakamoto, G. Matsunaga, and K. Itami. Investigations of impurity seeding and radiation control for long-pulse and high-density $\mathrm{H}$-mode plasmas in JT-60U. Nuclear Fusion, 49(11):115010, 2009.

[16] A. Kallenbach, M. Bernert, R. Dux, T. Eich, C. Giroud, A. Herrmann, J. W. Hughes, 
Numerical analyses of JT-60SA with tungsten divertor by COREDIV code

M. Lehnen, B. Lipschultz, A. Loarte, G. Maddison, F. Reimold, M. Reinke, J. Schweinzer, B. Sieglin, M. Wischmeier, S. Wolfe, ASDEX Upgrade Team, Alcator Team, and JET EFDA Contributors. Multi-machine comparisons of divertor heat flux mitigation by radiative cooling with nitrogen. In 24. IAEA Fusion Energy Conference. International Atomic Energy Agency (IAEA), 2012

[17] K. Shimizu, T. Takizuka, and H. Kawashima. Kinetic effect of thermal force on impurity transport: Simulation of JT-60SA divertor with integrated divertor code $\{\mathrm{SONIC}\}$. Journal of Nuclear Materials, 390-391:307-310, 2009.

[18] D.P. Coster, H. Klingshirn, L. Alves, V. Basiuk, J. Bizarro, J. Ferreira, A. Figueiredo, P. Huynh, F. Imbeaux, I. Ivanova-Stanik, D. Kalupin, G. Pereverzev, and R. Stankiewicz. Core-Edge Coupling: developments within the EFDA Task Force on Integrated Tokamak Modelling. In 39th EPS Conference on Plasma Physics, 16th International Congress on Plasma Physics, 2012.

[19] S. I. Braginskii. Transport Processes in a Plasma. Reviews of Plasma Physics, 1:205, 1965.

[20] R. Stankiewicz and R. Zagórski. Self-consistent modelling of the tokamak core and SOL plasma with the COREDIV code. Czechoslovak Journal of Physics, 52:D32-D37, 2002.

[21] M. Shimada, D. J. Campbell, M. Wakatani, H. Ninomiya, N. Ivanov, V. Mukhovatov, and the ITER Joint Central Team and Home Teams. Physics Basis of ITER-FEAT. In Fusion energy 2000. Fusion energy 1998 (2001 Edition). Proceedings. International Atomic Energy Agency (IAEA), 2001.

[22] WPSA Workshop on JT-60SA. 2015.

[23] Y.R. Martin, T. Takizuka, and the ITPA CDBM H-mode Threshold Database Working Group. Power requirement for accessing the H-mode in ITER. Journal of Physics: Conference Series, 123(1):012033, 2008.

[24] M. A. Makowski, D. Elder, T. K. Gray, B. LaBombard, C. J. Lasnier, A. W. Leonard, R. Maingi, T. H. Osborne, P. C. Stangeby, J. L. Terry, and J. Watkins. Analysis of a multi-machine database on divertor heat fluxes. Physics of Plasmas, 19(5), 2012. 